\title{
Single nucleotide polymorphism-based analysis of the genetic structure of Liangshan pig population
}

\author{
Bin Liu ${ }^{1,2, a}$, Linyuan Shen ${ }^{1,2, a}$, Zhixian Guo ${ }^{1,2}$, Mailing Gan ${ }^{1,2}$, Ying Chen ${ }^{3}$, Runling Yang ${ }^{4}$, Lili Niu ${ }^{1,2}$, \\ Dongmei Jiang ${ }^{1,2}$, Zhijun Zhong ${ }^{5}$, Xuewei $\mathrm{Li}^{1,2}$, Shunhua Zhang ${ }^{1,2, *}$, and Li Zhu ${ }^{1,2, *}$
}

\section{* Corresponding Authors: \\ Shunhua Zhang \\ Tel: +86-13982086205, Fax: +86-291010, \\ E-mail:363445986@qq.com \\ Li Zhu \\ Tel: +86-13982083385, Fax: +86-2291010, \\ E-mail: zhuli7508@163.com}

${ }^{1}$ College of Animal Science and Technology, Sichuan Agricultural University, Chengdu, Sichuan, 611130, China

2 Farm Animal Genetic Resources Exploration and Innovation Key Laboratory of Sichuan Province, Sichuan Agricultural University, Chengdu, Sichuan, 611130, China ${ }^{3}$ Sichuan Province General Station of Animal Husbandry, Chengdu 610066, China ${ }^{4}$ Agriculture and Rural Bureau of Mabian Yi Autonomous County, Mabian, 614600, China

${ }^{5}$ Sichuan Academy of Animal Sciences, Chengdu 610066, China

a These authors contributed equally to this work.

ORCID

Bin Liu

https://orcid.org/0000-0002-6526-7983 Linyuan Shen

https://orcid.org/0000-0001-6072-3268

Zhixian Guo

https://orcid.org/0000-0003-1425-8654 Mailing Gan

https://orcid.org/0000-0001-9900-3559 Ying Chen

https://orcid.org/0000-0002-1580-7259

Runling Yang

https://orcid.org/0000-0002-7837-0656 Lili Niu

https://orcid.org/0000-0002-9783-0945 Dongmei Jiang

https://orcid.org/0000-0001-9309-308X Zhijun Zhong

https://orcid.org/0000-0002-7640-9545 Xuewei Li

https://orcid.org/0000-0003-4560-5137 Shunhua Zhang

https://orcid.org/0000-0003-0569-0146 Li Zhu

https://orcid.org/0000-0001-7342-0880

Submitted Nov 18, 2019; Revised Jan 24, 2020; Accepted Apr 14, 2020
Objective: To conserve and utilize the genetic resources of a traditional Chinese indigenous pig breed, Liangshan pig, we assessed the genetic diversity, genetic structure, and genetic distance in this study.

Methods: We used 50K single nucleotide polymorphism (SNP) chip for SNP detection of 139 individuals in the Liangshan Pig Conservation Farm.

Results: The genetically closed conserved population consisted of five overlapping generations, and the total effective content of the population $(\mathrm{Ne})$ was 15 . The whole population was divided into five boar families and one non-boar family. Among them, the effective size of each generation subpopulation continuously decreased. However, the proportion of polymorphic markers $\left(\mathrm{P}_{\mathrm{N}}\right)$ first decreased and then increased. The average genetic distance of these 139 Liangshan pigs was $0.2823 \pm 0.0259$, and the average genetic distance of the 14 boars was $0.2723 \pm 0.0384$. Thus, it can be deduced that the genetic distance changed from generation to generation. In the conserved population, 983 runs of homozygosity $(\mathrm{ROH})$ were detected, and the majority of $\mathrm{ROH}(80 \%)$ were within $100 \mathrm{Mb}$. The inbreeding coefficient calculated based on $\mathrm{ROH}$ showed an average value of 0.026 for the whole population. In addition, the inbreeding coefficient of each generation subpopulation initially increased and then decreased. In the pedigree of the whole conserved population, the error rate of paternal information was more than $11.35 \%$ while the maternal information was more than $2.13 \%$.

Conclusion: This molecular study of the population genetic structure of Liangshan pig showed loss of genetic diversity during the closed cross-generation reproduction process. It is necessary to improve the mating plan or introduce new outside blood to ensure longterm preservation of Liangshan pig.

Keywords: Single Nucleotide Polymorphism (SNP) Chip; Liangshan Pig; Inbreeding Coefficient; Genetic Distance; Genetic Diversity

\section{INTRODUCTION}

According to the data (2004) from the Domestic Animal Diversity Information System (DAD-IS) and Food and Agricultural Organization (FAO), China produces one-third of the world's pig breeds [1]. Yet, the number of indigenous breeds has declined sharply in the past 20 years due to breeding selection for lean meat and high growth rate of foreign pig breeds. Liangshan pig is a small, traditional Chinese breed mainly distributed in the mountain areas of Yi Autonomous Prefecture with an altitude of 1,500 to 2,000 m [2]. It is well known for cold tolerance, crude feeding tolerance, and meat quality [3]. Due to a devastating outbreak of African swine fever in 2019, the number of Liangshan pigs declined. Therefore, it is important to study the genetic diversity and the changes in genetic structure of the Liangshan pig population to evaluate and protect China's abundant genetic resources. 
Due to its low cost in recent years, genome-wide genotyping has become beneficial when studying and researching the genetic information of livestock [4]. The genetic variation in some Chinese pig breeds has been extensively studied using high-density single nucleotide polymorphism (SNP) chips or whole genome sequencing [5]. Although the cost involved in genome-wide sequencing has greatly reduced, it is still relatively expensive. As a result, this hinders the use of genome-wide sequencing for the analysis of largescale samples. In this study, we use 50K SNP chip to analyze genetic diversity, genetic relationship, population structure, and inbreeding coefficient of Liangshan pigs in the conserved population farm.

\section{MATERIALS AND METHODS}

\section{Animal care}

All animal works were conducted according to the guidelines on the care and use of experimental animals established by the Ministry of Agriculture of China. The Animal Care and Ethics Committee of Sichuan Agricultural University specifically approved this study under Permit No. DKY-S2017 6906.

\section{Animals}

Ear tissues from 139 purebred Liangshan pigs were collected for DNA extraction from the Liangshan Pig Conservation Farm of Leshan, Sichuan province. After pedigree data query, all samples were divided into a total of five generation subpopulations (Supplementary Table S1).

\section{Single nucleotide polymorphism genotyping}

DNA was extracted from the ear tissues by phenol-chloroform extraction method [6], and the quality of DNA was detected by ultraviolet spectrophotometry (NanoDrop, 2000; Thermo Scientific, ShangHai, China) and gel electrophoresis (BIO-RAD \& DYPC-31BN, Newbio Gi-1, WuHan, China). The qualified 139 DNA samples were genotyped using “Zhongxin-I” Porcine Breeding Chip (Beijing Compass Agritechnology Co., Ltd., Beijing, China), which contains 51,315 SNPs. Quality control of genotype data was performed using PLINK (v1.90) software [7]. Only autosomal loci were used; SNPs with a minor allele frequency less than 0.05 and with a call rate less than $90 \%$ were eliminated.

\section{Analysis of genetic diversity}

Effective population size $(\mathrm{Ne})$ refers to the size of an ideal population with the same gene frequency variance or the same inbreeding coefficient increment (hybridity attenuation rate) as the actual population, which is estimated based on the level of linkage disequilibrium [8]. We used SNeP (v1.1) software to calculate $\mathrm{Ne}[8]$.
Polymorphic marker ratio $\left(\mathrm{P}_{\mathrm{N}}\right)$ refers to the proportion of polymorphic loci in the target population to the total number of loci. We first calculated the minimum allele frequency for each locus using PLINK (v1.90) [7] and then calculated $\mathrm{P}_{\mathrm{N}}$ using a self-programmed $R$ script [9]. We calculated $\mathrm{P}_{\mathrm{N}}$ using the formula as follows:

$$
P_{N}=\frac{M}{N}
$$

where $M$ is the number of sites that exhibit polymorphism and $N$ is the total number of sites.

Expected heterozygosity $(\mathrm{He})$ refers to the probability of heterozygosity at any one of the individuals in the population; observed heterozygosity (Ho) refers to the ratio of the number of individuals in a population where a locus is heterozygous to the total number of individuals. When the Ho is less than the He, we speculate that the population has experienced selection or inbreeding; if the Ho is more than the $\mathrm{He}$, the population may have introduced some other varieties. We used PLINK (v1.90) to calculate He and Ho [7].

\section{Calculation of genetic distance and genetic relationship}

We used PLINK (v1.90) to calculate idengtical by state (IBS) distances and $R$ script to build heat maps. IBS refers to the DNA fragment identical by descent in two or more individuals, and these DNA fragments have the same base sequence. IBS only considers the similarity of genetic markers or alleles between individuals, regardless of whether they come from the same ancestor or not. Therefore, there is no need for parental genotyping. The genetic distance based on IBS can still analyze the genetic relationship of the population without information on the pedigree or ancestral samples. We used $G$ matrix (v2) and $R$ to calculate kinship values and heat maps [10]. $G$ matrix is a genomic relationship matrix constructed with whole genome markers. Since the pedigree information of a conserved population is usually not recorded, $G$ matrix is suitable for calculating the genetic relationship.

\section{Analysis of population structure}

After quality control of the genotype data, 36,592 SNP loci were used to analyze the population structure. The population structure is mainly clustered by the neighboring method (neighbor-joining $[\mathrm{NJ}]$ ) and based on IBS distance matrix [11]. Based on the analysis, we can roughly infer which Liangshan pig individuals are close in blood in general, samples originated from the same family group. We used PLINK (v1.90) for population structure analysis [7].

Inbreeding coefficient analysis

Widespread in all populations, runs of homozygosity (ROH) 
are contiguous segments in an individual genome due to complete transfer of a homologous haplotype from a parent to a progeny. The length and frequency of $\mathrm{ROH}$ can reflect the group history. A long $\mathrm{ROH}$ indicates recent inbreeding, while a short $\mathrm{ROH}$ indicates ancient inbreeding. First, the length of ROH in each sample was calculated by PLINK (v1.90) [7]. Then the ratio of the total length of the $\mathrm{ROH}$ fragment to the total length of autosomal genome was calculated to get the coefficient of inbreeding based on $\mathrm{ROH}$ [12] using the following formula:

$$
F_{R O H} \frac{\sum_{k} \operatorname{Length}\left(\mathrm{ROH}_{k}\right)}{L}
$$

where $k$ is the number of $\mathrm{ROH}$ in the individual and $L$ is the length of the autosomal genome of the species (porcine v10.2 version of the genome, autosomal length is approximately 2,450,713 Kb).

\section{Pedigree accuracy analysis}

Pedigree accuracy was analyzed using Mendelian errors based on paired individuals (individual-parent, individual-mother) with genotypes in the pedigree [13]. Mendelian error indicates which of an individual's allele is not from any of its biological parents, and the Mendelian error rate identifies the proportion of markers that have made a mistake in the calculation. If a pair of individuals with a Mendel error rate greater than $1 \%$ is discovered in the pedigree, then the pedigree is considered wrong and will be found in other homosexual samples that provide genotypes. If a sample with a Mendel error rate below $1 \%$ is not found, the individual is considered missing. If multiple samples with a Mendel error rate below $1 \%$ are found, the sample with the smallest Mendel error rate (born earlier to offspring) is selected as the true parent of the individual.

\section{RESULTS}

Single nucleotide polymorphism characteristics

A total of 51,315 SNP loci were detected from 139 samples. PLINK (v1.90) [7] was used to remove loci on sex chromosomes, and SNP genotyping data of 44,739 SNP loci were used for the subsequent analysis.

\section{Pedigree accuracy of the conserved population of Liangshan pigs}

Information on a total of 26 sires and 19 dams were available in the pedigree record of 139 Liangshan pigs (Table 1). After genotypic analysis, 4 wrong sire records were corrected. We found that the information on 12 sires and 3 dams were erroneous; however, their true parents were not detected via genotyping. A total of 97 sires and 117 dams were excluded from the experimental group tested by the chip. Therefore, it was impossible to judge the accuracy of the information. Altogether, in the conserved population, the pedigree error rate of sire information was more than $11.51 \%$, and the error rate of dam information was more than $2.16 \%$.

\section{Genetic diversity of the Liangshan pigs}

The Ne of these 139 purebred pigs of Liangshan Pig Conservation Farm was 15.00 and the proportion of $\mathrm{P}_{\mathrm{N}}$ was 0.8393 . We observed significant differences in population effective content $(\mathrm{Ne})$ among the different generations. The sample size of the F5 generation subpopulation was 10 and the effective population content was 2.31. The sample size of the F3 generation subpopulation was 41 and the effective population content was 7.89. Lowest proportion of $\mathrm{P}_{\mathrm{N}}(0.8060)$ was in the F4 generation subpopulation and highest (0.8393) was in the F5 generation subpopulation. In the conserved population and all these five generations, the $\mathrm{He}(0.3478)$ was less than the Ho (0.3551), which suggests some other species mixed in the population (Table 2).

\section{Genetic distance of the conserved population of Liangshan pigs}

We measured an IBS distance that varied from 0.1261 to 0.3442 for the conserved population of Liangshan pigs and the generation subpopulations. We also determined that the overall population average genetic distance to be $0.2823 \pm$ 0.0259. It showed that the average genetic distance among Liangshan pigs is far apart, and the variation of the population is quite large. The genetic distance among 14 breeding boars ranged from 0.1659 to 0.3136 , and the average genetic distance was $0.2723 \pm 0.0384$. The genetic distances of all the five generation subpopulations ranged between 0.2765 and 0.2882 . The genetic distance of the F5 generation subpopulation was the lowest $(0.2765 \pm 0.0253)$ and the F4 generation subpopulation was the highest $(0.2882 \pm 0.0279)$ (Table 3$)$.

Table 1. Rectifying results of Liangshan pig pedigree

\begin{tabular}{lcc}
\hline Result type & Number of sires & Number of dams \\
\hline No genotype was provided. & 97 & 117 \\
The original pedigree did not match and the real parents were not detected. & 12 & 3 \\
The original pedigree did not match and the real parents were detected. & 4 & 0 \\
Matching with the original pedigree & 26 & 19 \\
\hline
\end{tabular}


Table 2. Genetic diversity parameters of Liangshan pigs by generations

\begin{tabular}{lcccc}
\hline Generation & $\mathbf{N e}$ & $\mathbf{P}_{\mathbf{N}}$ & $\mathbf{H e}$ & Ho \\
\hline F1 & 6.70 & 0.8339 & 0.3526 & 0.3677 \\
F2 & 7.78 & 0.8339 & 0.3477 & 0.3604 \\
F3 & 7.89 & 0.8146 & 0.3507 & 0.3583 \\
F4 & 4.88 & 0.8060 & 0.3563 & 0.3697 \\
F5 & 2.31 & 0.8393 & 0.3327 & 0.3595 \\
F & 15.00 & 0.8393 & 0.3478 & 0.3551 \\
\hline
\end{tabular}

$\mathrm{Ne}$, effective population content; $\mathrm{P}_{\mathrm{N}}$, the proportion of single nucleotide polymorphisms (SNPs) that displayed polymorphism in 44,739 SNPs selected from the 50K panel; He, expected heterozygosity; Ho, observed heterozygosity.

Visualization results of IBS distance matrix and $\boldsymbol{G}$ matrix of the conserved population and the different generation subpopulations showed that most of the individuals have moderate
Table 3. Identity by state genetic distance of Liangshan pigs

\begin{tabular}{lcccc}
\hline Generation & Max & Min & Average & $\begin{array}{c}\text { Standard } \\
\text { deviation }\end{array}$ \\
\hline F1 & 0.3442 & 01372 & 0.2826 & 0.0264 \\
F2 & 0.3248 & 0.1565 & 02797 & 0.0218 \\
F3 & 0.3363 & 0.1261 & 0.2822 & 0.0271 \\
F4 & 0.3395 & 0.1409 & 0.2882 & 0.0279 \\
F5 & 0.3052 & 0.1953 & 0.2765 & 0.0253 \\
F & 0.3442 & 0.1261 & 0.2823 & 0.0259 \\
Boar & 0.3136 & 0.1659 & 0.2723 & 0.0384 \\
\hline
\end{tabular}

Max, maximum value; Min, minimum value.

genetic relationship and some have high genetic relationship. These findings indicated a potential inbreeding trend among these individuals (Figures 1,2), and therefore, we need to pay special attention to their mating plan.

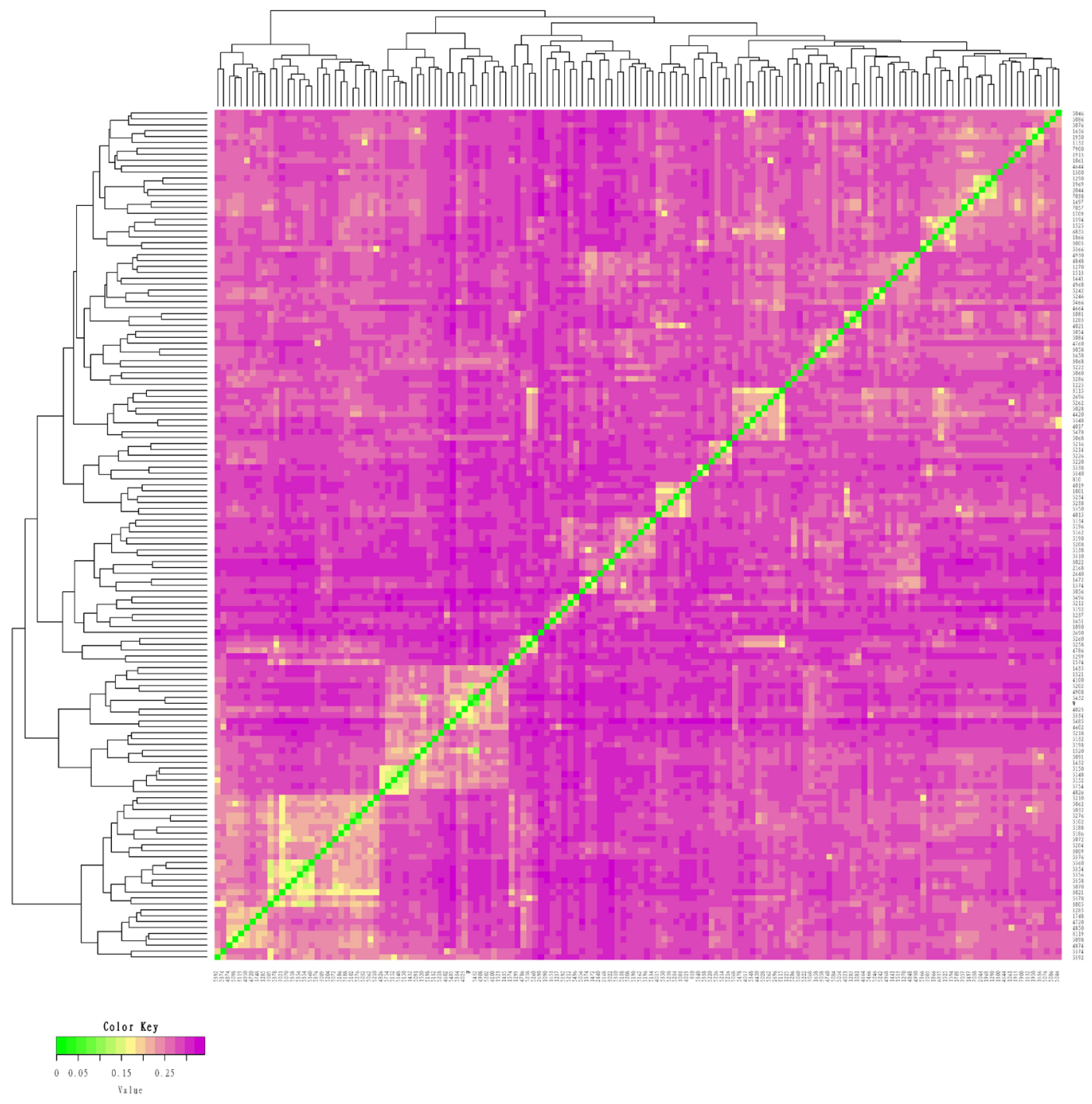

Figure 1. Identity by state (IBS) distance matrix of the conserved population of Liangshan pigs. Using Plink software and R package to make IBS heat map. Each small square in IBS distance matrix represents the genetic distance value between two pairs from the first sample to the last sample. The larger the value is, the closer it is to red, that is, the larger the genetic distance between two individuals is, that is, the two individuals are not similar, and vice versa. 


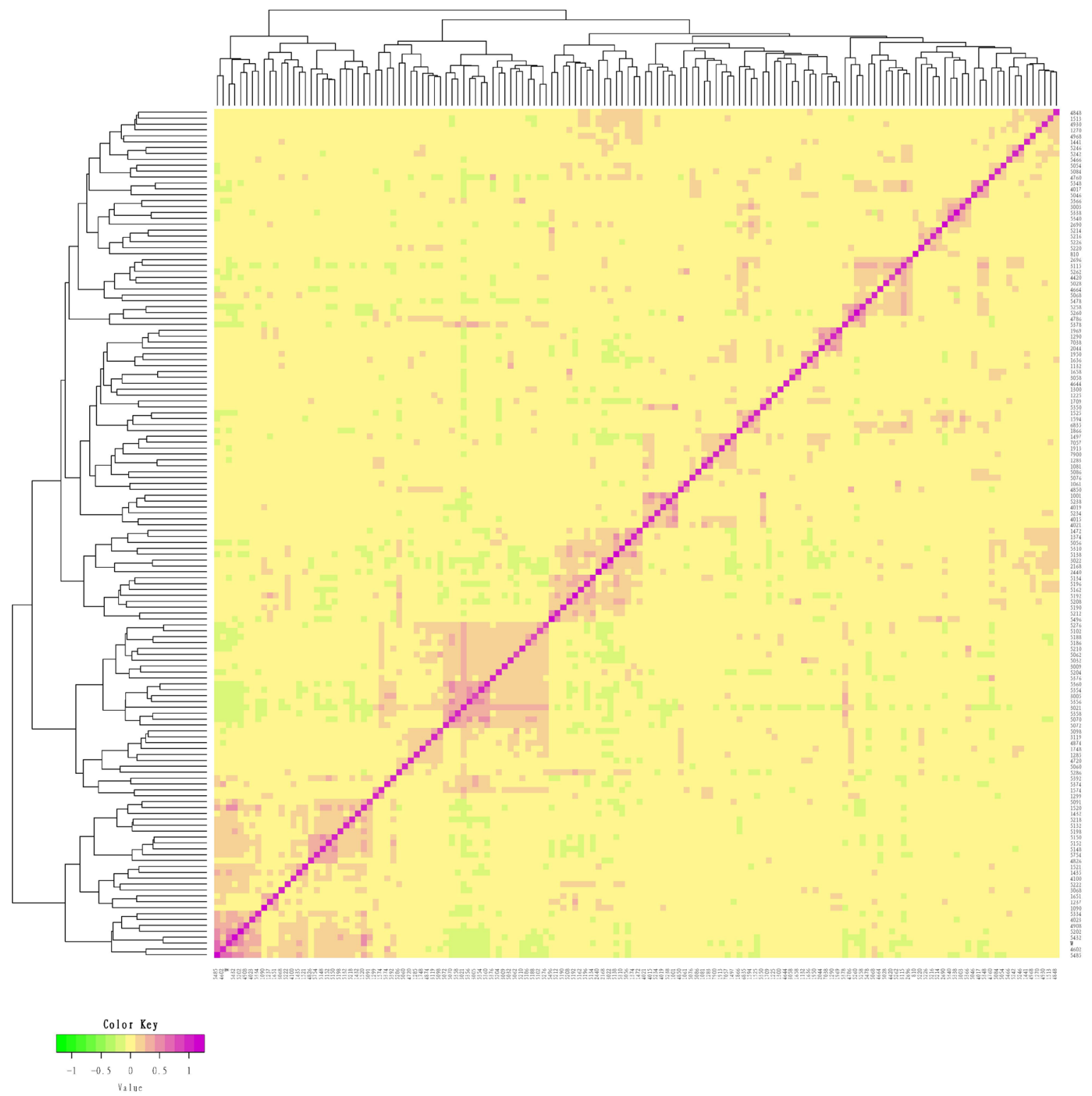

Figure 2. $\mathbf{G}$ matrix of the conserved population of Liangshan pigs. Using $\mathbf{G}$ matrix software and R package to make $\mathbf{G}$ matrix heat map. Each small square represents the value of the relationship between two pairs from the first sample to the last sample. The larger the value is, the closer it is to red, that is, the closer the relationship between two individuals is.

\section{Population family genetic structure of Liangshan pigs} Due to the importance of boars in the conservation process, this study combined the $\boldsymbol{G}$ matrix and IBS distance matrix to analyze the phylogenetic tree of all 14 boars in the population using the $\mathrm{NJ}$ method. The existing boars were divided into five different consanguinity families. Samples labeled with the same color in the evolutionary tree were evaluated as the same family, and the same consanguinity family branches were divided into the second or more inner clustering units in the evolutionary tree (Figure 3). Based on NJ tree and the relationship matrix, combined with the relationship between sows and boars of different families, all individuals in the conserved population were eventually divided into five large families. In addition to the five families, 49 sows were genetically less related to any of the tested breeding boars (genetic relationship coefficient less than 0.1 ). Therefore, they were classified as another family (Table 4, Figure 4).

\section{Inbreeding coefficient of the Liangshan pigs based on runs of homozygosity}

A total of $983 \mathrm{ROH}$ were detected in these 139 Liangshan pigs. The total length of $\mathrm{ROH}$ of each individual ranged between $2.76 \mathrm{Mb}$ and $522.51 \mathrm{Mb}$, and the average $\mathrm{ROH}$ length of the conserved population was $63.24 \pm 101.92 \mathrm{Mb}$. Liangshan pig with $\mathrm{ROH}$ less than $100 \mathrm{Mb}$ has the largest number of individuals ( $86.11 \%$ of the protected population; Figure 5) 


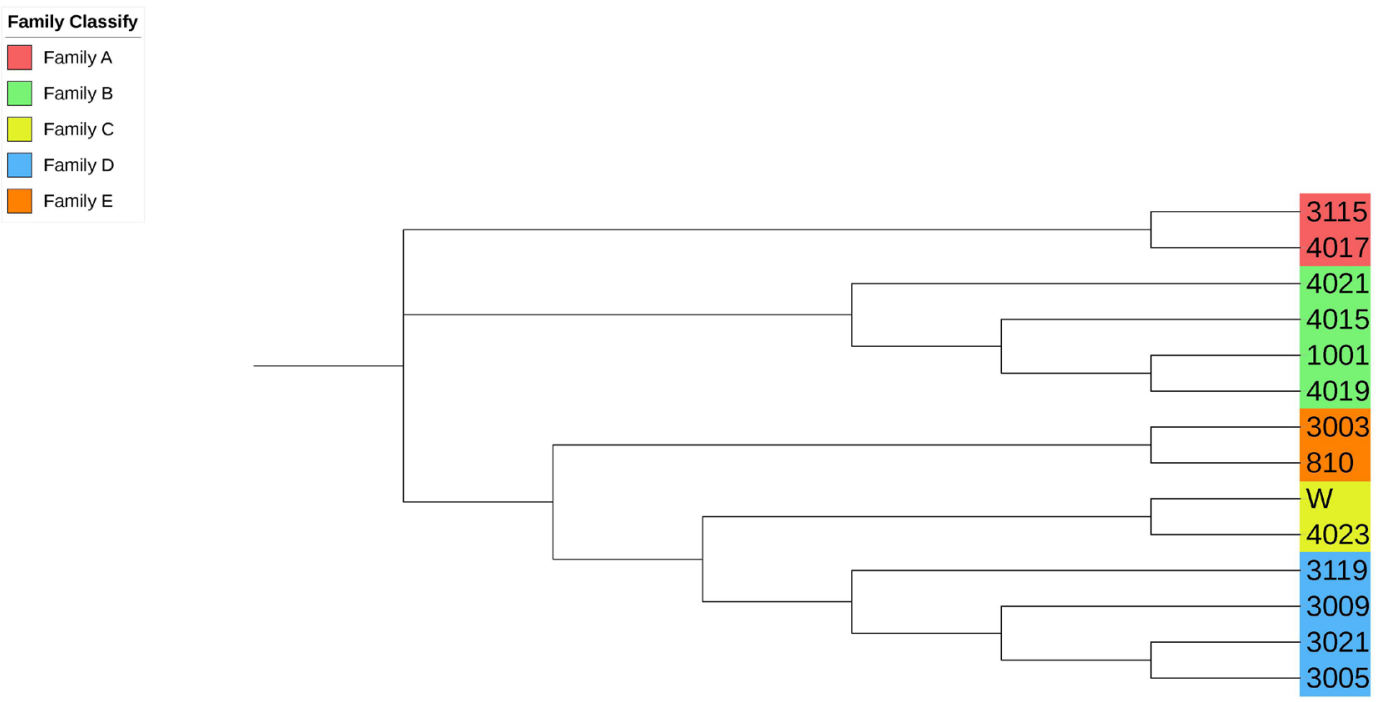

Figure 3. Evolutionary tree of boar samples in the conserved population. Samples labeled with the same color are evaluated as the same family. All boars include 5 families.

The total number of $\mathrm{ROH}$ in each Liangshan pig varied between 0 and 33 (an average of 7.07 \pm 6.48 ), and the individuals with 0 to $5 \mathrm{ROH}$ was the largest in the conserved population (Figure 6). Overall, the number of $\mathrm{ROH}$ positively correlated with chromosome length. We obtained the inbreeding coefficient value of each individual through $\mathrm{ROH}$ statistics. In the genetically closed conserved population of Liangshan pigs, the average inbreeding coefficient of the conserved population was 0.026 . However, the average inbreeding coefficient of each generation subpopulation changed significantly. Among the different generation subpopulations, the highest inbreeding coefficient was 0.035 in the third generation and the lowest was 0.018 in the fifth generation (Figure 7).

\section{DISCUSSION}

Pedigree accuracy analysis

In the breeding work, data recording errors or defects are

Table 4. Consanguinity family construction in the conserved population

\begin{tabular}{lcc}
\hline Family & Gender & Quantity \\
\hline Family A & Boar & 2 \\
& Sow & 17 \\
Family B & Boar & 4 \\
& Sow & 11 \\
Family C & Boar & 2 \\
& Sow & 21 \\
Family D & Boar & 4 \\
& Sow & 32 \\
Family E & Boar & 2 \\
& Sow & 8 \\
Other & Sow & 49 \\
\hline
\end{tabular}

inevitable, which also decrease the accuracy and integrity of pedigrees. In indigenous pig conservation farms, the working conditions are normally very tough. So far no database has been established and genetic information relies on the handwriting in the records. It is impossible to exclude errors in genealogical records caused by the negligence of staff members or existing unrecorded genealogical information. According to statistics, the average pedigree error rate in animal and tree breeding population can reach up to $10 \%$ $[14,15]$. The genome homozygosity index based on whole genomic markers can reflect the inbreeding degree of individuals. It has more application in revealing the actual inbreeding level and inbreeding genetic effect of the population [16]. In the absence of pedigree data, the correction of pedigree inbreeding coefficient with genome inbreeding coefficient is a more accurate tool to measure individual inbreeding degree [17]. Correcting pedigree errors can improve best linear unbiased prediction-breeding value predictions and improve heritability estimates [16], which can effectively protect the Liangshan pig population. Through our analysis, we discovered an error rate greater than $11.35 \%$ in the paternal information and more than $2.13 \%$ in maternal information on Liangshan pigs, thus meaning that the error rate in paternal information was more than that in maternal information. This may be because the maternal information normally comes from daily farm records on reproduction and mating; however, there are many times in the process of artificial insemination. This may further lead to errors in paternal information. Therefore, we need to strengthen the accuracy of pedigree records in the subsequent breeding works on Liangshan pigs. 


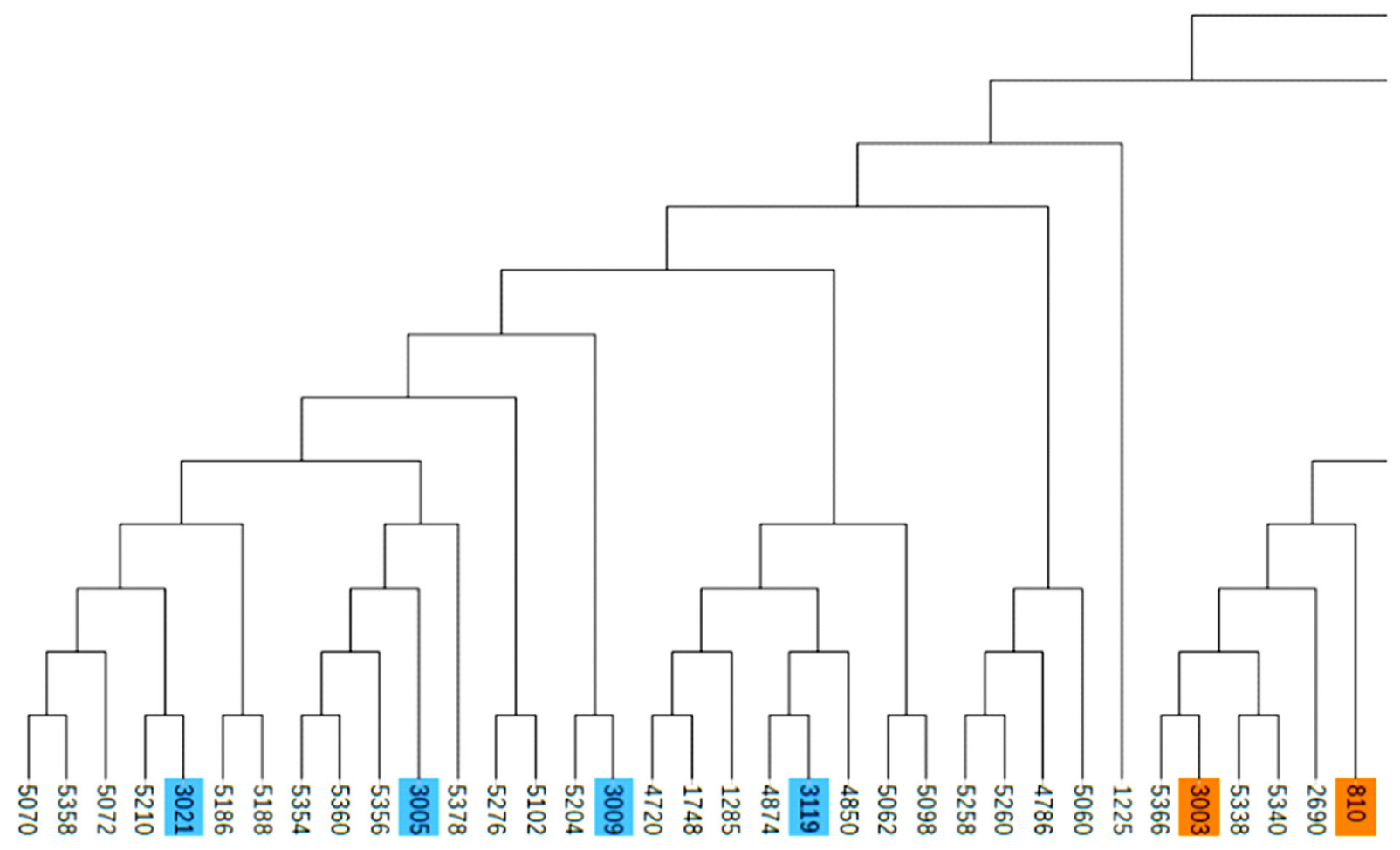

Figure 4. Partial screenshot of the phylogenetic tree. Samples labeled with the same color are evaluated as the same family.

\section{Genetic diversity}

Using genotype data to assess the $\mathrm{Ne}$ of the current population is a very important research hotspot in conservation genetics $[18,19]$. High-density SNP panels improve the accuracy in population parameter estimation, including $\mathrm{Ne}$ [20]. Our analysis revealed that the conserved population of Liangshan pigs has remained genetically closed and kept reproducing from the formation of the basic group till the fifth generation. Now, the whole conserved population consists of five overlapping generations. We recorded an $\mathrm{Ne}$ of 15 (the whole conservation population), and with increasing generations, the $\mathrm{Ne}$ of each generation subpopulation showed a significant variation. The $\mathrm{Ne}$ of the fifth generation was only 2.31 , which may be because only 10 samples were available in this generation. Using the same method, Shin et al [21] estimated the effective population content of a Landrace

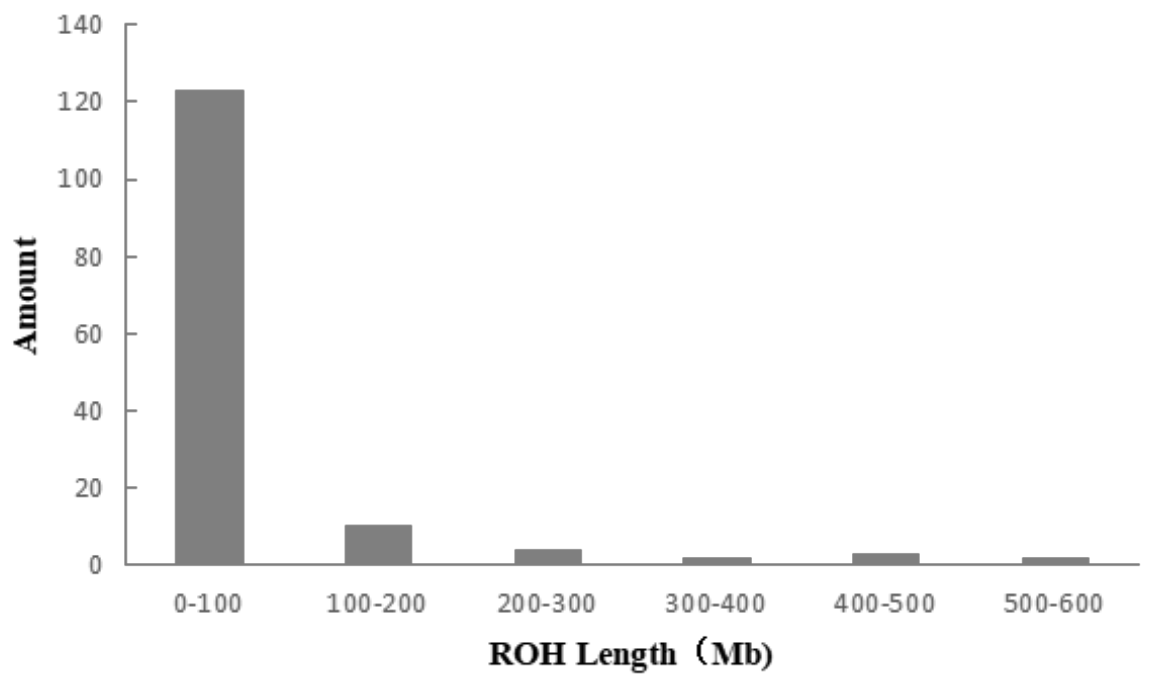

Figure 5. Distribution of ROH lengths in the individuals of Liangshan pigs. Taking 50 SNPs as windows, moving 5 SNPs at a time and calculating the length of $\mathrm{ROH}$. The length of $\mathrm{ROH}$ in 0 to $100 \mathrm{Mb}$ is the largest in Liangshan pigs. ROH, runs of homozygosity; SNP, single nucleotide polymorphism. 


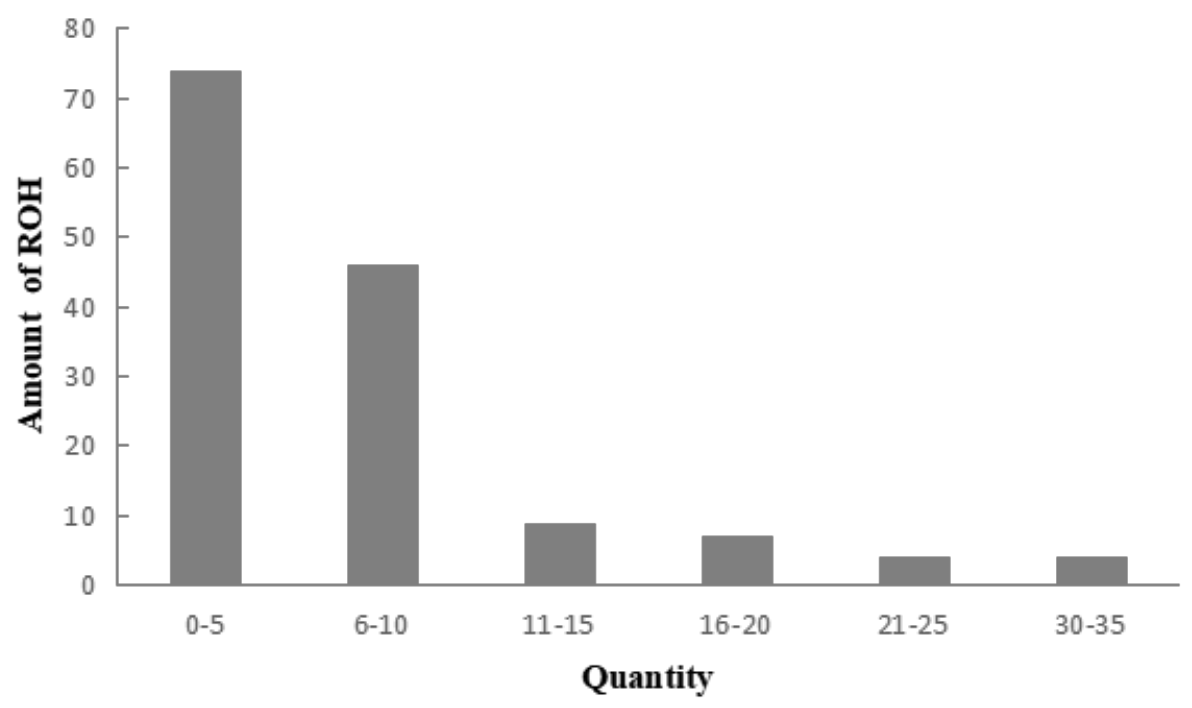

Figure 6. Distribution of runs of homozygosity $(\mathrm{ROH})$ in the individuals of Liangshan pigs.

pig population (1,128 individuals) as 92.27. Due to the small size of the population and their unfavorable natural habitat (mountain areas), Liangshan pigs has experienced an extinction crisis. By 2010, the conserved population was established, but the Ne of Liangshan pigs still remains low after five generations of closed reproduction due to limited independent blood families of the basic group. This is currently a major problem that the vast majority of indigenous livestock and poultry breed in China face. Therefore, in the conservation of indigenous pig breeds, we need to actively introduce new consanguinity, especially new boar consanguinity, and pay special attention to selection and mating schemes to prevent the loss of independent consanguinity in the alternation of generations. Comprehensive genetic diversity analysis showed that the SNP polymorphism $\left(\mathrm{P}_{\mathrm{N}}\right)$ kept varying among different gene ratio subpopulations. The SNP polymorphism $\left(\mathrm{P}_{\mathrm{N}}\right)$ of the whole conserved population was 0.8393 , which was less than that of other Chinese indigenous breeds (Bihu, Chalu, Chun'an, Jinghua, Shengxianhua, Lanxihua, and Jiaxinghei) [22]. The Ho was more than the He for all the generation subpopulations and for the whole conserved population, which indicates that the conserved population contained mixedbreeds and need to be further purified by eliminating impure individuals. In the conserved population of Liangshan pigs, the genetic diversity decreased after generations of closed reproduction. In addition, the heterozygosity of the population (both $\mathrm{He}$ and $\mathrm{Ho}$ ) was low, which may be due to inevitable inbreeding or loss of less productive families. This is also

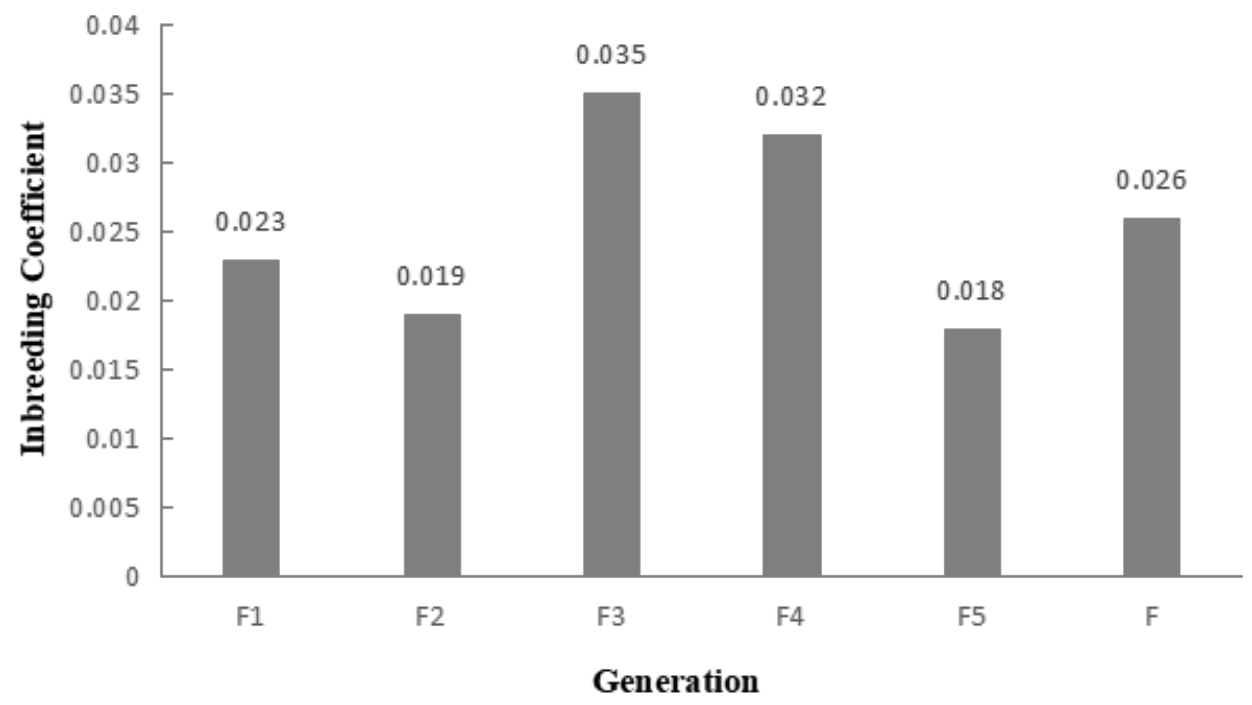

Figure 7. Average inbreeding coefficient of the conserved population and different generation subpopulations. By calculating the proportion of the total length of runs of homozygosity $(\mathrm{ROH})$ fragment to the total length of autosomal genome, the coefficient of inbreeding based on ROH was obtained. 
common in other small-sized Chinese indigenous livestock and poultry breeds [14].

\section{Genetic distance of the conserved population and the generation subpopulations}

In order to protect the population from the influence of exotic varieties, most of the conserved livestock populations in China remain genetically closed. Therefore, it is important to study genetic distance, genetic relationship, and genetic structure of the conserved population, especially the multigeneration overlapping population, to maintain sustainable development [23]. IBS showed that the genetic distance of the whole conserved population and of the 14 breeding boars was 0.2823 and 0.2723 , respectively. After five generations of closed reproduction, the genetic distance of the subpopulations slightly changed, and the fifth generation subpopulation had the lowest genetic distance (0.2765). These findings indicate that the conserved population experienced a selection pressure during generation alternation, which changed the genetic structure of the closed conserved population.

\section{Analysis of population genetic structure}

In order to analyze the degree of genetic background difference of the whole population, we used the NJ method and clustered the samples based on IBS distance matrix [5]. In the $\mathrm{NJ}$ tree, the whole conserved population was divided into five different families with boars and a family without boars. At present, the number and inbreeding degree of boars from each family are not balanced in the whole population. In three families, there were only two boars. Therefore, in the subsequent conservation process, we need to pay special attention to the number of pigs in each family during mating plans to avoid the loss of blood lines and maintain a balanced family structure. After identifying the effective blood relationship of breeding boars by SNP chip, we can use this as a guide to select for reproduction and to construct frozen semen banks of different breeds. This will help reduce the cost of genetic resources and improve the efficiency of conservation.

\section{Analysis of inbreeding coefficient based on runs of homozygosity}

Chromosome fragments containing homozygous SNP genotypes are used to infer possible haploids inherited by the same individual, and $\mathrm{ROH}$ (Froh) is used to estimate the inbreeding coefficient of the genome [24]. In addition, a long $\mathrm{ROH}$ indicates a more recent genetic relationship, a short $\mathrm{ROH}$ denotes ancient inbreeding, and a greater quantity of $\mathrm{ROH}$ fragments implies a higher possibility of inbreeding in the family [25]. At present, the number of individuals with $\mathrm{ROH}$ length within $100 \mathrm{MB}$ is the largest in the whole population, and especially the number of $\mathrm{ROH}$ within 5 is the largest. This may be due to the different generations and selection strategies used by the conserved farm, as well as the different degrees of selection pressure on the traits of these lines [26]. Bosse et al [27] found that $\mathrm{ROH}$ larger than $5 \mathrm{Mb}$ could be detected using a $60 \mathrm{~K}$ SNP chip, and $\mathrm{ROH}$ larger than $5 \mathrm{Mb}$ were as accurate as the whole genome sequencing. In this experiment, we determined $0 \mathrm{Mb}$ to be the shortest $\mathrm{ROH}$ of Liangshan pigs and $63.24 \mathrm{Mb}$ as the average length, which proves the accuracy and validity of our results. In addition, our results showed that $86.11 \%$ of $\mathrm{ROH}$ were shorter than $100 \mathrm{Mb}$, which suggests ancient inbreeding. Due to the limited population size of the indigenous pig breeds and the relatively closed operation system, after reproducing for successive generations and the aggravation of overlapping generations, the inbreeding coefficient of the population kept increasing and the genetic structure of the conserved population changed dramatically. Our analysis revealed that the inbreeding coefficient of the genetically closed conserved population of Liangshan pigs kept increasing, and the inbreeding coefficient of the third generation subpopulation was the highest (3.5\%). Due to the introduction of certain boars after that, the inbreeding coefficient decreased. Now, the inbreeding coefficient of the whole conserved population remains at $2.6 \%$.

\section{CONCLUSION}

By studying the population genetic structure of Liangshan pigs at the molecular level, we found that the effective population content and the genetic diversity of the conserved population were low during the process of subculture, which led to a change in the genetic structure of the population to a certain extent. This variation in genetic structure may be caused by other breeds that got mixed in the population. To protect LiangShan pigs, it is necessary to further purify the population and increase the matching strength or import foreign consanguinity to ensure long-term preservation of genetic resources. Further study on the genetic structure of the local protected population in China at the molecular level will effectively help to protect these excellent local varieties.

\section{CONFLICT OF INTEREST}

We certify that there is no conflict of interest with any financial organization regarding the material discussed in the manuscript.

\section{ACKNOWLEDGMENTS}

This study was supported by the Sichuan Science and Technology Support Program (No. 2016NYZ0050; No. SCSZTD -3-008), the National Natural Science Foundation of China 
(No. 31530073), and the earmarked fund for China Agriculture Research System (No. CARS-36-05B).

\section{REFERENCES}

1. Fang $\mathrm{M}, \mathrm{Hu} \mathrm{X}$, Jiang $\mathrm{T}$, et al. The phylogeny of Chinese indigenous pig breeds inferred from microsatellite markers. Anim Genet 2005;36:7-13. https://doi.org/10.1111/j.1365-2052. 2004.01234.x

2. Luo J, Lei H, Shen L, et al. Estimation of growth curves and suitable slaughter weight of the Liangshan pig. Asian-Australas J Anim Sci 2015;28:1252-8. https://doi.org/10.5713/ajas.15. 0010

3. Jones GF. Genetic aspects of domestication, common breeds and their origin. In: Rothschild MF, Ruvinsky A, editors. The genetics of the pig. Wallingford, UK: CAB International; 1998. pp. 17-50.

4. Ai H, Yang B, Li J, Xie X, Chen H, Ren J. Population history and genomic signatures for high-altitude adaptation in Tibetan pigs. BMC Genomics 2014;15:834. https://doi.org/10.1186/ 1471-2164-15-834

5. Ai H, Huang L, Ren J. Genetic diversity, linkage disequilibrium and selection signatures in Chinese and Western pigs revealed by genome-wide SNP markers. PLoS One 2013;8:e56001. https://doi.org/10.1371/journal.pone.0056001

6. Chomczynski P, Sacchi N. The single-step method of RNA isolation by acid guanidinium thiocyanate-phenol-chloroform extraction: twenty-something years on. Nat Protoc 2006;1: 581-5. https://doi.org/10.1038/nprot.2006.83

7. Purcell S, Neale B, Todd-Brown K, et al. PLINK: a tool set for whole-genome association and population-based linkage analyses. Am J Hum Genet 2007;81:559-75. https:/doi.org/ 10.1086/519795

8. Barbato M, Orozco-terWengel P, Tapio M, Bruford MW. $S N e P$ : a tool to estimate trends in recent effective population size trajectories using genome-wide SNP data. Front Genet 2015;6:109. https://doi.org/10.3389/fgene.2015.00109

9. Chan BKC. Data analysis using R programming. In: Biostatistics for epidemiology and public health using RAdv. New York, USA: Springer Publishing Company; 2015. pp. 81-154. https://doi.org/10.1891/9780826110268.0003

10. VanRaden PM. Efficient methods to compute genomic predictions. J Dairy Sci 2008;91:4414-23. https://doi.org/10.3168/ jds.2007-0980

11. Kumar S, Stecher G, Li M, Knyaz C, Tamura K. MEGA X: molecular evolutionary genetics analysis across computing platforms. Mol Biol Evol 2018;35:1547-9. https://doi.org/10. 1093/molbev/msy096

12. Silió L, Rodríguez MC, Fernández A, et al. Measuring inbreeding and inbreeding depression on pig growth from pedigree or SNP-derived metrics. J Anim Breed Genet 2013;130:34960. https://doi.org/10.1111/jbg.12031
13.Wiggans GR, VanRaden PM, Bacheller LR, et al. Selection and management of DNA markers for use in genomic evaluation. J Dairy Sci 2010;93:2287-92. https://doi.org/10.3168/ jds.2009-2773

14. SanCristobal M, Chevalet C, Haley CS, et al. Genetic diversity within and between European pig breeds using microsatellite markers. Anim Genet 2006;37:189-98. https://doi. org/10.1111/j.1365-2052.2005.01385.x

15. Visscher PM, Woolliams JA, Smith D, Williams JL. Estimation of pedigree errors in the UK dairy population using microsatellite markers and the impact on selection. J Dairy Sci 2002;85:2368-75. https://doi.org/10.3168/jds.S0022-0302 (02)74317-8

16. Munoz PR, Resende MFR, Huber DA, et al. Genomic relationship matrix for correcting pedigree errors in breeding populations: impact on genetic parameters and genomic selection accuracy. Crop Sci 2014;54:1115-23. https://doi.org/10.2135/ cropsci2012.12.0673

17. Powell JE, Visscher PM, Goddard ME. Reconciling the analysis of IBD and IBS in complex trait studies. Nat Rev Genet 2010;11:800-5. https://doi.org/10.1038/nrg2865

18. Waples RS. Making sense of genetic estimates of effective population size. Mol Ecol 2016;25:4689-91. https://doi.org/ $10.1111 / \mathrm{mec} .13814$

19. Waples RK, Larson WA, Waples RS. Estimating contemporary effective population size in non-model species using linkage disequilibrium across thousands of loci. Heredity 2016;117: 233-40. https://doi.org/10.1038/hdy.2016.60

20. Wang L, Sørensen P, Janss L, Ostersen T, Edwards D. Genomewide and local pattern of linkage disequilibrium and persistence of phase for 3 Danish pig breeds. BMC Genet 2013; 14:115. https://doi.org/10.1186/1471-2156-14-115

21.Shin D, Kim SH, Park J, Lee HK, Song KD. Extent of linkage disequilibrium and effective population size of the Landrace population in Korea. Asian-Australas J Anim Sci 2018;31: 1078-87. https://doi.org/10.5713/ajas.17.0237

22. Chen J, Peng J, Xiao Q, et al. The genetic diversity and population structures of indigenous pig breeds in Zhejiang Province revealed by GGRS sequencing. Anim Genet 2018;49:36-42. https://doi.org/10.1111/age.12625

23. Wang X, Cao HH, Geng SM, Li HB. Genetic diversity of 10 indigenous pig breeds in China by using microsatellite markers. Asian-Australas J Anim Sci 2004;17:1219-22. https:// doi.org/10.5713/ajas.2004.1219

24. Bjelland DW, Weigel KA, Vukasinovic N, Nkrumah JD. Evaluation of inbreeding depression in Holstein cattle using wholegenome SNP markers and alternative measures of genomic inbreeding. J Dairy Sci 2013;96:4697-706. https://doi.org/10. 3168/jds.2012-6435

25. Kirin M, McQuillan R, Franklin CS, Campbell H, McKeigue PM, Wilson JF. Genomic runs of homozygosity record population history and consanguinity. PLoS One 2010;5:e13996. 
https://doi.org/10.1371/journal.pone.0013996

26.Zanella R, Peixoto JO, Cardoso FF, et al. Genetic diversity analysis of two commercial breeds of pigs using genomic and pedigree data. Genet Sel Evol 2016;48:24. https://doi. org/10.1186/s12711-016-0203-3
27. Bosse M, Megens HJ, Madsen O, et al. Regions of homozygosity in the porcine genome: consequence of demography and the recombination landscape. PLoS Genet 2012;8: e1003100. https://doi.org/10.1371/journal.pgen.1003100 